\title{
Quantitative Atomistic Simulations of Reactive and Non-Reactive Processes
}

\author{
Markus Meuwly*
}

\begin{abstract}
The interpretation of physico-chemical observables in terms of atomic motions is one of the primary objectives of atomistic simulations. Trajectories from a molecular simulation contain much valuable information about the relationship between motion of the atoms and physical observables related to them, provided that the interactions used to generate the trajectories are of sufficiently high quality. On the other hand, many experimental observables are averages over a large number of physical realizations of the system. Thus, a statistically large number of trajectories needs to be generated and analyzed in order to provide a meaningful basis for comparison with and interpretation of experiments. The preferred computational approach which allows such extensive averaging while retaining the quantitative aspects of the intermolecular interactions are accurate force field-based molecular dynamics simulations. This contribution provides an overview of our group's current technological improvements in force field technology and its application to fundamental physico-chemical questions.
\end{abstract}

Keywords: Computational spectroscopy · Force fields · Molecular dynamics simulations · Multipoles · Reaction dynamics

In the physical sciences simulations provide a third approach - next to experiment and theory - to characterize and understand a wide range of phenomena. For chemistry and chemical physics in particular, atomistic simulations are paramount in providing insights into the energetics and dynamics of complex systems, such as proteins, chemical reactivity, solvation dynamics or spectroscopy.

The use of atomistic simulations in reaction dynamics has been established for almost 50 years when molecular dynamics (MD) simulations were used to better understand the $\mathrm{H}_{2}+\mathrm{H}$ reaction dynamics. ${ }^{[1]}$ Since then, MD simulations have become an important complement to investigate complex systems at atomic resolution. The major driving forces behind this development are i) the increase in computational power, ii) the improvement of algorithms and energy functions and iii) the more direct interaction between experiment and simulation. It is the latter that will eventu-

\footnotetext{
${ }^{*}$ Correspondence: Prof. M. Meuwly

Department of Chemistry

University of Basel

Klingelbergstrasse 80

$\mathrm{CH}-4056$ Basel

Tel: +41612673821

E-mail:m.meuwly@unibas.ch
}

ally let this field mature to a degree which allows quantitative and ideally predictive work to be carried out which must be the ultimate goal of atomistic simulations.

In the present contribution I will summarize our efforts to item ii) above. More specifically, our group is concerned with improved and widely applicable energy functions for spectroscopic and thermodynamic applications and the development of computational methods to follow chemical reactions in the gas phase and in solution.

\section{Quantitative Intermolecular Interactions}

Within the framework of atomistic simulations, intermolecular interactions are described by an empirical energy function ('force field') which is based on a ball-andspring model for the bonded interactions (such as bonds, valence angles, dihedrals) and Coulomb and van der Waals interactions for the nonbonded interactions. [2] This is in contrast to explicit solutions of the electronic Schrödinger equation for fixed nuclear positions which provides depending on the quantum chemical method and basis set used - accurate energetics. However, for repeated evaluations $\left(10^{6}\right.$ to $10^{8}$ times for a typical MD simulation) such methods cannot be routinely and efficiently used. A typical force field is written in parametrized form as $V_{\text {tot }}=V_{\text {bond }}+$ $V_{\text {valence }}+V_{\text {dihe }}+V_{\text {elstat }}+V_{\text {vdW }}$ where each of the terms is separately parametrized:

The first three terms comprise 'bonded' interactions while the latter two describe the 'non-bonded' ones. In these

$$
\begin{gathered}
V_{\text {bond }}=\sum K_{b}\left(r-r_{e}\right)^{2} \\
V_{\text {valence }}=\sum K_{\theta}\left(\theta-\theta_{e}\right)^{2} \\
V_{\text {dihe }}=\sum K_{\phi}(1-\cos (n \phi-\delta)) \\
V_{\text {elstat }}=\frac{1}{4 \pi \varepsilon_{0}} \sum \frac{q_{i} q_{j}}{r_{i j}} \\
V_{v d W}=\sum \varepsilon_{i j}\left[\left(\frac{R_{\min , i j}}{r_{i j}}\right)^{12}-\left(\frac{R_{\min , i j}}{r_{i j}}\right)^{6}\right]
\end{gathered}
$$

expressions, $K$ are force constants associated with the particular type of interaction, $r$ and $\theta_{e}$ are equilibrium geometries, $n$ is the periodicity of the dihedral and $\delta$ is the phase which determines the location of the maximum. The sums for the bonded terms are carried out over all atoms involved. The parameters in the bonded interactions are typically fitted to reproduce experimental data from structural work (X-ray, NMR, microwave) or spectroscopy (IR, Raman).[3]

Nonbonded interactions include electrostatic and van der Waals terms where the sums include all nonbonded atom pairs. $q_{i}$ and $q_{j}$ are the partial charges of the atoms $i$ and $j$ involved and $\varepsilon_{0}$ is the vacuum dielectric constant. For the van der Waals terms, the potential energy is expressed as a Lennard-Jones potential with well depth $\varepsilon_{i j}=\sqrt{\varepsilon_{i} \varepsilon_{j}}$ and range $\mathrm{R}_{\min , i j}=\left(\mathrm{R}_{\min , i}+\mathrm{R}_{\min , j}\right) / 2$ at the Lennard-Jones minimum. This interaction captures long range dispersion $\left(\propto-r^{-6}\right)$ and exchange repulsion $\left(\propto r^{-12}\right)$ where the power of the latter is chosen for convenience. 
Over the past few years, our group has been primarily involved in providing more physically motivated treatments of the electrostatics. This includes specifically multipolar (MTP) interactions which are capable of capturing finer details related to lone-pairs (e.g. nitric oxide), $\pi$-systems, or the $\sigma$-hole. ${ }^{[4-8]}$ Originally, the use of MTPs was motivated by the desire to correctly describe the spectroscopy of small ligands in electrostatically demanding environments, such as proteins or water ices. ${ }^{[4,9,10]}$ For this, fluctuating MTPs were employed for which the atom-centered multipoles vary as a function of the bond length of the diatomic. Such an approach was found to quantitatively describe the splitting, red shift and line shape for infrared spectra. ${ }^{[4,9,11]}$ A signature application for such a MTP force field was the investigation of the $1 \mathrm{~d}$ - and $2 \mathrm{~d}$-spectroscopy of $\mathrm{CN}^{-}$in water. Using a force field optimized for the vibrational relaxation of cyanide in water, the spectroscopic properties could be faithfully reproduced. ${ }^{[11,12]}$ This contrasts with a point-charge-based model which was unable to describe the experimentally determined line shape and structural dynamics amenable to 2d-infrared spectroscopy, see Fig. 1.[13] This finding corroborated earlier, more qualitative work which already noted that a distributed charge model with four nuclear charges was required for this problem. ${ }^{[14]}$ Finally, it was also possible to correctly describe the solvation free energy for $\mathrm{CN}^{-}$in water using the same parametrization. ${ }^{[15]}$ Hence, vibrational relaxation, 1d-, 2d-spectroscopy and solvation free energy of an anion in solution can be quantitatively described by using a refined MTP model in atomistic simulations.

\section{Reactive Molecular Dynamics}

Following the energetics and dynamics of chemical reactions is of fundamental importance in all branches of chemistry and biology. Experimentally, reaction mechanisms often need to be inferred from phenomenological models because simultaneous determination of the physical trajectories of the atoms involved and their energetics is typically not possible. Hence, computational methods play an essential role in elucidating, at atomic resolution, the possible reaction pathways. The most rigorous computational treatment of such processes uses quantum methods for the electronic structure and nuclear dynamics. As this is usually impractical due to the computational effort involved, the nuclear motion (often of a subset of the atoms involved) is sometimes treated quantum mechanically while using a parametrized potential energy surface (PES). This has been successfully done for a number of topical

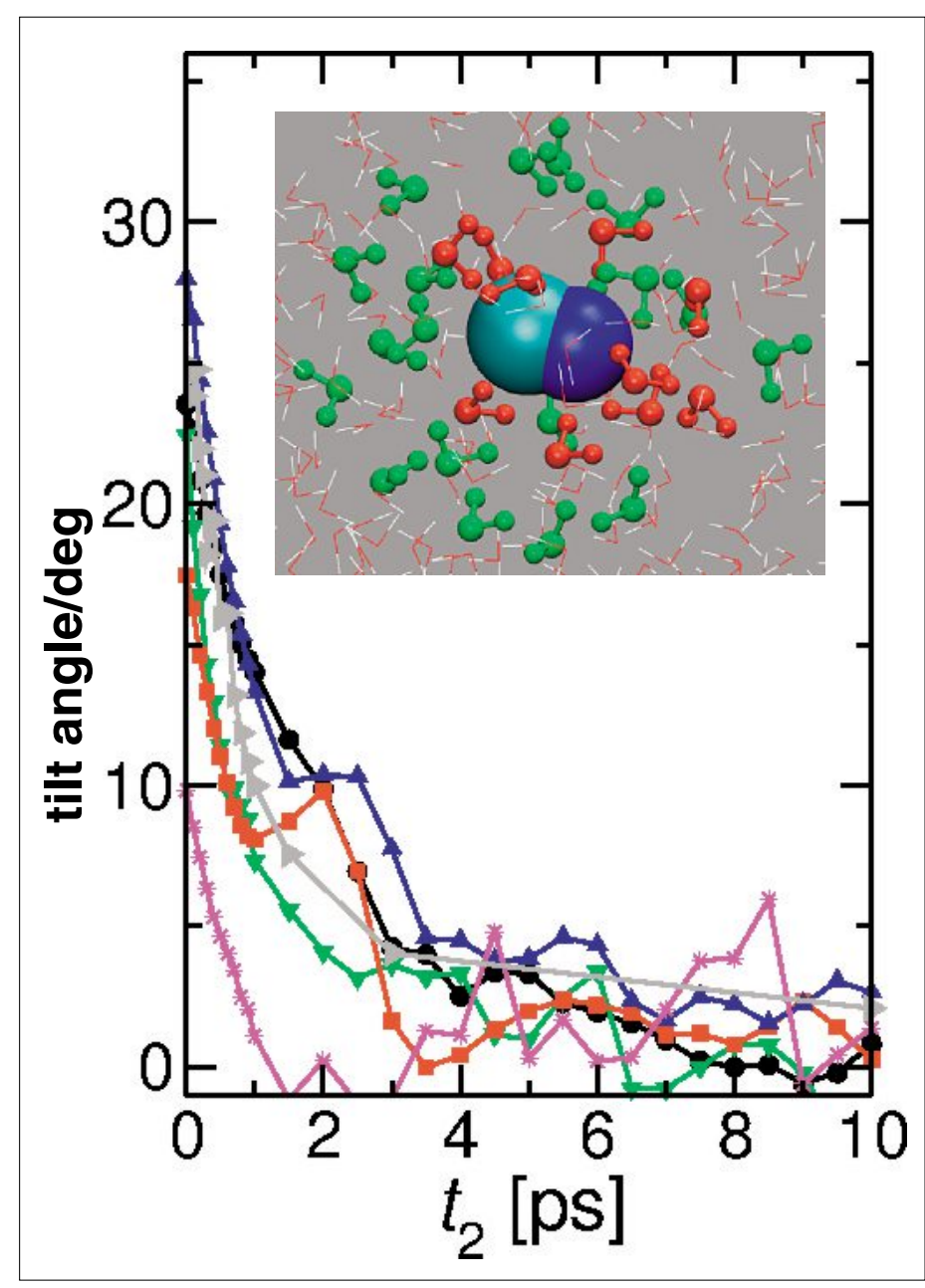

Fig. 1. Comparison of tilt angles for $\mathrm{CN}^{-}$in water as a function of mixing time $t_{2}$ determined from the raw data. Black, cyan, green, red, and blue traces are from different MTP models. Results from PC simulations are in magenta. For comparison tilt angles from experiment ${ }^{[14]}$ are shown in gray in both panels. Figure adapted from ref. [11].

systems. ${ }^{[16-21]}$ As a thermal rate constant is an ensemble average over a large number of initial conditions, a statistically significant number of trajectories needs to be run and analyzed. Therefore, approaches which treat the nuclear dynamics at the classical level have become an attractive and often meaningful alternative. Such simulations can, under favorable circumstances, be run by resorting to quantum mechanical (QM) ${ }^{[22-24]}$ or mixed quantum/ molecular mechanics (QM/MM) treatments. ${ }^{[25-27]}$ Alternatively, if empirical and fully-dimensional force fields of sufficient accuracy can be parametrized, they offer a viable means to exhaustively sample phase space from which meaningful rate parameters can be determined. ${ }^{[28-33]}$

One of the methods developed by us is adiabatic reactive MD (ARMD). Two variants exist for this: a time-based ${ }^{[29,34]}$ and an energy-based switching scheme. ${ }^{[35]}$ In both approaches, multiple force fields are parametrized, one for each 'state' involved. In the simplest case, one force field describes the reactant and a second one the product state. ${ }^{[32,36-38]}$ However, additional physically meaningful states (e.g. intermediates) can be included in order to more completely describe the physical situation. Within time-based switching, the different force fields are joined through $t$-depen- dent switching functions during crossing. The occurrence of a crossing is found by continuously comparing the energies of the different states for the momentary configuration. ${ }^{[29]}$ As the resulting Hamiltonian is time-dependent, rigorous ( $N V E$ ) simulations are not possible. This is usually not a limitation for reactions in the condensed phase. ${ }^{[36,38]}$ However, for gas-phase reactions, final state analysis becomes meaningless. [32,35]

Multi-Surface-ARMD (MS-ARMD) is an energy-based switching method which yields energy-conserving trajectories. ${ }^{[35]}$ It is particularly suited for situations in which details of the barrier crossing are of interest and has so far been applied to gas-phase reactions. ${ }^{[35,39,40]}$ The mixing of the asymptotic PESs involves coordinatedependent weights

$w(\mathbf{x})=\exp \left(-\frac{V_{i}(\mathbf{x})-V_{\min }(\mathbf{x})}{\Delta V}\right)$

in which $V_{i}(\mathbf{x})$ is any of the $i$ PESs, $V_{\min }(\mathbf{x})$ is the lowest of the $i$ PESs for the given conformation $\mathbf{x}$ and $\Delta V$ is a characteristic energy scale which determines how smoothly the switching between the PESs occurs. $\Delta V$ plays an analogous role as the switching time in time-based switching, see Fig. 2. 


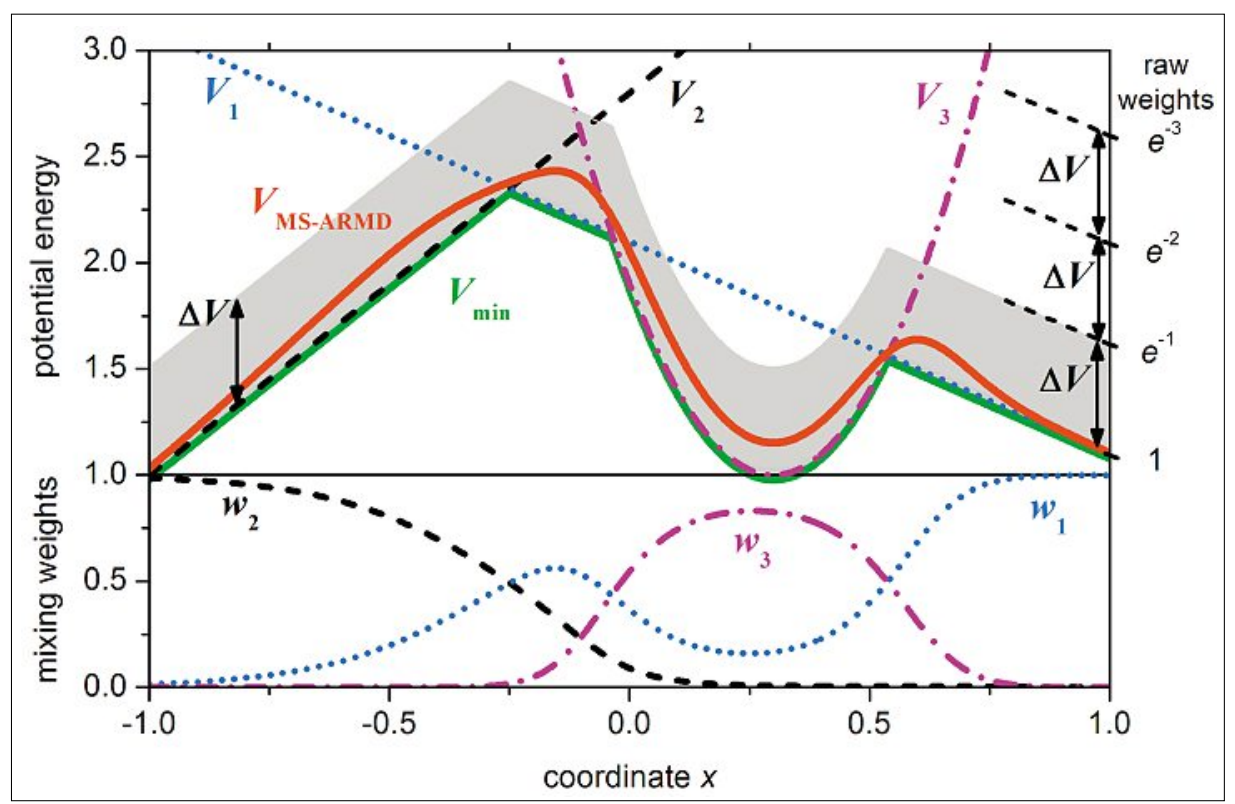

Fig. 2. Illustration of MS-ARMD in one dimension $(x)$ for three surfaces $\left(V_{1}\right.$ : blue dotted, $V_{2}$ : black dashed, $V_{3}$ : magenta dot-dashed). The effective surface $\left(V_{\text {MS-ARMD }}\right.$ : red solid) always follows the lowest-energy surface (green solid), except for regions where several surfaces are close in energy (within a few times $\Delta V=0: 5$ ). Here, the algorithm switches smoothly among them by varying their weights $\left(w_{1}, w_{2}\right.$, and $w_{3}$; see lower panel) as a function of $\mathrm{x}$. All quantities are in arbitrary units. Figure adapted from ref. [35].

As an example, the results from MSARMD simulations on the rotational excitation of $\mathrm{N}_{2}{ }^{+}$in collisions with $\mathrm{N}_{2}$ are briefly summarized.[39] Experimentally, fully quantum cooled $\mathrm{N}_{2}^{+}$ions in their ground vibrational and rotational state were collided with $\mathrm{N}_{2}$ molecules from a cold beam at $8 \mathrm{~K}$. After the collision, fragment $\mathrm{N}_{2}{ }^{+}$ions with rotational angular momentum up to $N=2$ were observed. Consequently, translationto-rotation excitation took place during the charge-transfer reaction. A microscopic understanding from the experiments alone was not possible. Atomistic simulations on a fully-dimensional, permutation-invariant PES were carried out to this end. The relevant states involved the $\mathrm{N}_{2}{ }^{+}+\mathrm{N}_{2}$ entrance channel, the $\mathrm{N}_{4}^{+}$bound state $(\approx 1.3 \mathrm{eV}$ stabilized relative to the asymptote) and the $\mathrm{N}_{2}{ }^{+}+\mathrm{N}_{2}$ exit channel. As no third body is present, the $\mathrm{N}_{4}^{+}$ion must decay after a finite lifetime which was on average $100 \mathrm{ps}$ from the simulations. The quality of the PES for the bound state compared to the reference UCCSD/cc-pVTZ calculations was $0.06 \mathrm{eV}$ and $0.013 \mathrm{eV}$ for the unbound state. Averaging several hundred reactive trajectories yielded an average probability for CT of $50 \%$ which is what is statistically expected. The second-order rate coefficient as determined from the scattering cross section $\sigma_{\text {tot }}=243 \pm 19 \mathrm{a}_{0}^{2}$ and the relative velocity of $787 \mathrm{~m} / \mathrm{s}$ was $k=$ $(5.36 \pm 0.42) \times 10^{10} \mathrm{~cm}^{3} \mathrm{~s}^{-1}$ which compares favorably with previous experimental results of $k=4.24 \times 10^{10}, k=6.6 \times 10^{10}$, and $k=5.0 \times 10^{10} \mathrm{~cm}^{3} \mathrm{~s}^{-1} \cdot{ }^{[41-43]}$ Such validation simulations are essential to determine how meaningful the computational model is.
Armed with this insight, detailed analysis of the trajectories showed that the origin of the rotational excitation in the product channel originates from intensive bending vibrations in the bound $\mathrm{N}_{4}^{+}$state which, upon dissociation, imprints on the leaving fragments. It is noteworthy that even for such a highly quantum system classical molecular dynamics simulations can provide a realistic computational framework which allows interpretation of complex experimental data.

\section{Linear and Multidimensional Spectroscopy}

Computational spectroscopy is another field in which atomistic simulations can a) be meaningful complements to and b) provide invaluable insights into experimentally characterized systems. For linear, one-dimensional spectroscopies the Fourier-Transform of the dipole moment autocorrelation function provides a direct link to the experimentally measured spectrum. Typically, simulations on the nanosecond time scale are sufficient to converge the spectra. ${ }^{[4,9,44-47]}$ For multidimensional spectroscopies, considerably more sampling is required to converge the underlying frequency-frequency correlation function $C(t)=<\delta \omega(0) \delta \omega(t)>$, where $\delta \omega(t)=$ $\omega(t)-<\omega>$ is the instantaneous deviation of the transition frequency $\omega(t)$ from the mean $\langle\omega\rangle$. ${ }^{[11]}$ This naturally precludes the use of mixed quantum mechanical/molecular mechanics (QM/MM) approaches. For $\mathrm{CN}^{-}$in water a direct comparison of the tilt angle, which is one of the direct experimental observables, determined from simulations with PC and MTP charge models establishes that more physics-based force fields are required for realistically following the solvation dynamics. ${ }^{[11,13]}$

\section{Solvation Dynamics}

Atomistic simulations are also valuable to characterize the rapid dynamics of the solvent around a solute. One situation in which this is of fundamental importance is the relaxation of vibrational energy of an excited chromophore. For cyanide solvated in $\mathrm{H}_{2} \mathrm{O}$ and $\mathrm{D}_{2} \mathrm{O}$ experiment found $\mathrm{CN}^{-}$vibrational relaxation times to differ by a factor 3 in heavy water compared to regular water. ${ }^{[5]}$ Based on a resonance model, the decay of energy into the solvent was related to inter- and intramolecular degrees of freedom. However, direct proof of this mechanism, involving the water bending vibration and the $z$-libration was only given by simulations. ${ }^{[48]}$ Using an MTP force field it was established that the vibrational energy of the cyanide relaxes into these two degrees of freedom whereas all other vibrations remain thermalized after excitation.

In another study, photoexcitation of a Ru-tris-bipyridine complex was found to lead to restructuring of the water shell surrounding the metal-complex on the picosecond time scale. ${ }^{[49]}$ This agrees with recent time-resolved X-ray experiments on $\mathrm{Fe}$ (bpy) ${ }_{3}$ complexes in which it was found that upon excitation the number of water molecules reduces by two on the picosecond time scale. ${ }^{[50]}$ Corresponding simulations for the Fe-complex, also using the VALBOND-Trans (VBT) force field, confirm this finding. ${ }^{[51]}$ The simulations employ the VBT force field ${ }^{[52]}$ which is an extension of the VALBOND force field. VALBOND, developed by Landis and coworkers, ${ }^{[53-55]}$ is based on valence bond theory, where hybrid orbital strength functions are used for the molecular mechanics. Following foundations laid by Pauling ${ }^{[56,57]}$ who examined chemical bonding from the perspective of valence bond theory, VALBOND aimed at correctly capturing bending potentials over a wide range of angular distortions which primarily determine the shape of molecules. Together with molecular mechanics for proton transfer (MMPT), ${ }^{[58]}$ VBT was also successfully used to follow the structural dynamics and associated time scales around platinum complexes relevant to hydroformylation reactions. ${ }^{[59,60]}$ Specifically, all experimentally observed time scales for the reorganization involving the Pt-complex could be quantitatively interpreted based on the simulation results. This highlights the prominent role of atomistic 
simulations in understanding and providing the necessary complementary information to experiment.

\section{Summary and Outlook}

The research described in the present contribution summarizes our efforts in generalizing and improving molecular simulation technology for better characterizing the spatio-temporal evolution of complex molecular systems. The two main focal areas of particular interest are: a) the improvement of molecular mechanics force fields (MTP, VALBOND, MMPT) and $b$ ) following chemical reactions with empirical force fields (ARMD, MS-ARMD, MMPT). All these methods are implemented in the most recent versions of CHARMM.[61]

Atomistic simulations have extended the toolbox of computational and physical chemists over the past 40 years. This was recognized by the award of the 2013 Nobel Prize to Karplus, Levitt and Warshel. One essential step the field has to take now is to become predictive. For this, three major aims need to be accomplished - some of which have achieved to a remarkable degree. First, sampling needs to be further improved. This is not so much of an issue anymore for processes on the sub-nanosecond time scale, for which hundreds and thousands of independent trajectories can be run and provide statistically meaningful averages. Second, the quality of the force fields needs to be continuously improved. Third, analysis of large amounts of simulation data needs to be automatized and robust ways for data management, analysis and interpretation are required. Finally, the tools available need to be improved such that experimentalists are in a position to confidently and successfully employ the tools described here. This requires in particular automatized interfaces for fitting tasks $^{[62]}$ and easy-to-use graphical platforms for simulation tasks.

\section{Acknowledgement}

I thank all coworkers, past and present, for their contribution to the work presented here and reflected in the citations. We are grateful for the financial support granted by the Schweizerischer Nationalfonds through funding of projects Nr. 200021-117810 and through the NCCR MUST and to the University of Basel for continued financial and infrastructure support.

Received: May 25, 2014
[1] M. Karplus, R. Sharma, R. Porter, J. Chem. Phys. 1964, 40, 2033.

[2] S. Lifson, A. Warshel, J. Chem. Phys. 1968, 49, 5116.

[3] A. D. MacKerell, Jr., D. Bashford, M. Bellott, R. L. Dunbrack, Jr., J. D. Evanseck, M. J. Field, S. Fischer, J. Gao, H. Guo, S. Ha, D. JosephMcCarthy, L. Kuchnir, K. Kuczera, F. T. K. Lau, C. Mattos, S. Michnick, T. Ngo, D. T. Nguyen, B. Prodhom, W. E. Reiher, III, B. Roux, M. Schlenkrich, J. C. Smith, R. Stote, J. E. Straub, M. Watanabe, J. Wiorkiewicz-Kuczera, D. Yin, M. Karplus, J. Phys. Chem. B 1998, 102, 3586.

[4] N. Plattner, M. Meuwly, Biophys. J. 2008, 94 , 2505.

[5] M. W. Lee, M. Meuwly, J. Phys. Chem. B 2012, 116,4154

[6] C. Kramer, P. Gedeck, M. Meuwly, J. Comp. Chem. 2012, 33, 1673.

[7] C. Kramer, P. Gedeck, M. Meuwly, J. Chem. Theo. Comp. 2013, 9, 1499.

[8] T. Bereau, C. Kramer, M. Meuwly, J. Chem. Theo. Comp. 2013, 9, 5450

[9] D. R. Nutt, M. Meuwly, Biophys. J. 2003, 85, 3612.

[10] N. Plattner, M. Meuwly, Chem. Phys. Chem. 2008, 9, 1271 .

[11] M. W. Lee, J. K. Carr, M. Göllner, P. Hamm, M. Meuwly, J. Chem. Phys. 2013, 139, 054506.

[12] M. W. Lee, M. Meuwly, J. Phys. Chem. A 2011, $115,5053$.

[13] M. Kozínski, S. Garrett-Roe, P. Hamm, Chem. Phys. 2007, 341, 5 .

[14] R. Rey, J. T. Hynes, J. Chem. Phys. 1998, 108, 142

[15] M. W. Lee, M. Meuwly, PhysChemChemPhys 2013, 15, 20303.

[16] J. Hwang, A. Warshel, J. Am. Chem. Soc. 1996 , 118,11745 .

[17] K. Hinsen, B. Roux, J. Chem. Phys. 1997, 106, 3567.

[18] M. Pavese, S. Chawla, D. Lu, J. Lobaugh, G. A. Voth, J. Chem. Phys. 1997, 107, 7428.

[19] W. H. Miller, Y. Zhao, M. Ceotto, S. Yang, $J$. Chem. Phys. 2003, 119, 1329.

[20] K. F. Wong, J. L. Sonnenberg, F. Paesani, T. Yamamoto, J. Vanicek, W. Zhang, H. B. Schlegel, D. A. Case, T. E. Cheatham, III, W. H. Miller, G. A. Voth, J. Chem. Theo. Comp. 2010, $6,2566$.

[21] J. Huang, M. Buchowiecki, T. Nagy, J. Vanicek, M. Meuwly, PhysChemChemPhys 2014, 16, 204.

[22] R. Car, M. Parrinello, Phys. Rev. Lett. 1985, 55, 2471.

[23] J. Herbert, M. Head-Gordon, PhysChem ChemPhys 2005, 7, 3269

[24] A. M. N. Niklasson, C. J. Tymczak, M Challacombe, Phys. Rev. Lett. 2006, 97.

[25] A. Warshel, M. Levitt, J. Mol. Biol. 1976, 103, 227.

[26] M. J. Field, P. A. Bash, M. Karplus, J. Comp. Chem. 1990, 11, 700.

[27] P. A. Bash, M. J. Field, M. Karplus, J. Am. Chem. Soc. 1987, 109, 8092

[28] A. Warshel, R. M. Weiss, J. Am. Chem. Soc. 1980, 102, 6218

[29] J. Danielsson, M. Meuwly, J. Chem. Theo. Comp. 2008, 4, 1083.

[30] S. Mishra, M. Meuwly, J. Am. Chem. Soc. 2010, 132, 2968

[31] C. A. Rivera, N. Winter, R. V. Harper, I. Benjamin, S. E. Bradforth, PhysChemChemPhys 2011, 13, 8269 .
[32] J. Yosa, M. Meuwly, J. Phys. Chem. A 2011, 115,14350 .

[33] K. Farah, F. Mueller-Plathe, M. C. Boehm, Chem. Phys. Chem. 2012, 13, 1127.

[34] D. Nutt, M. Meuwly, Biophys. J. 2006, 90, 1191.

[35] T. Nagy, J. Yosa, M. Meuwly, J. Chem. Theo. Comp. 2014, 10, 1366.

[36] S. Mishra, M. Meuwly, J. Am. Chem. Soc. 2010, 132, 2968.

[37] S. Lutz, M. Meuwly, Chem. Phys. Chem. 2012, 13,305 .

[38] K. Nienhaus, S. Lutz, M. Meuwly, G. U. Nienhaus, Chem. Eur. J. 2013, 19, 3558

[39] X. Tong, T. Nagy, J. Y. Reyes, M. Germann, M. Meuwly, S. Willitsch. Chem. Phys. Lett. 2012, 547,1

[40] J. Yosa, T. Nagy, M. Meuwly, J. Phys. Chem. B 2014, submitted.

[41] M. Frost, S. Kato, V. Bierbaum, S. Leone, $J$. Chem. Phys. 1994, 100, 6359.

[42] N. Adams, D. Smith, Astrophys. J. 1981, 247, L123.

[43] T. McMahon, P. Miasek, J. Beauchamp, Intern. J. Mass Spect. Ion Proc. 1976, 21, 63.

[44] D. R. Nutt, M. Meuwly, Proc. Natl. Acad. Sci. 2004, 101, 5998 .

[45] K. Nienhaus, S. Lutz, M. Meuwly, G. U. Nienhaus, ChemPhysChem 2010, 11, 119 .

[46] S. Lutz, M. Meuwly, Faraday Discuss. Chem. Soc. 2011, 150, 375 .

[47] P. Hamm, M. Lim, R. M. Hochstrasser, J. Chem. Phys. 1997, 107, 10523.

[48] M. W. Lee, M. Meuwly, J. Phys. Chem. A 2011, 115, 5053.

[49] J. J. Szymczak, F. D. Hofmann, M. Meuwly, PhysChemChemPhys 2013, 15, 6268

[50] K. Haldrup, G. Vanko,W. Gawelda, A. Galler, G. Doumy, A.M. March, E. P. Kanter, A. Bordage, A. Dohn, T. B. van Driel, K. S. Kjaer, H. T. Lemke, S. E. Canton, J. Uhlig, V. Sundstrom, L. Young, S. H. Southworth, M.M. Nielsen, C. Bressler, J. Phys. Chem. A 2012, 116, 9878.

[51] A. S. Das, F. Hofmann, V. Solomon, M. Meuwly, in preparation, 2014

[52] I. Tubert-Broman, M. Schmid, M. Meuwly, $J$. Chem. Theo. Comp. 2009, 5, 530.

[53] D. M. Root, C. R. Landis, T. Cleveland, J. Am. Chem. Soc. 1993, 115, 4201

[54] T. Cleveland, C. G. Landis, J. Am. Chem. Soc. 1996, 118,6020

[55] C. R. Landis, T. Cleveland, T. K. Firman, J. Am. Chem. Soc. 1998, 120, 2641.

[56] L. Pauling, Proc. Natl. Acad. Sci. 1928, 14, 359.

[57] L. Pauling, J. Am. Chem. Soc. 1931, 53, 1367.

[58] S. Lammers, S. Lutz, M. Meuwly, J. Comp. Chem. 2008, 29, 1048

[59] U. Gellrich, J. Huang, W. Seiche, M. Keller, M. Meuwly, B. Breit, J. Am. Chem. Soc. 2011, 133, 964.

[60] J. Huang, D. Haeussinger, U. Gellrich, W. Seiche, B. Breit, M. Meuwly, J. Phys. Chem. B 2012, 116, 14406

[61] B. R. Brooks, C. L. Brooks, III, A. D. Mackerell, Jr., L. Nilsson, R. J. Petrella, B. Roux, Y. Won, G. Archontis, C. Bartels, S. Boresch, A. Caflisch, L. Caves, Q. Cui, A. R. Dinner, M. Feig, S. Fischer, J. Gao, M. Hodoscek, W. Im, K. Kuczera, T. Lazaridis, J. Ma, V. Ovchinnikov, E. Paci, R. W. Pastor, C. B. Post, J. Z. Pu, M. Schaefer, B. Tidor, R. M. Venable, H. L. Woodcock, X. Wu, W. Yang, D. M. York, M. Karplus, J. Comp. Chem. 2009, 30, 1545 .

[62] M. Devereux, M. Meuwly, J. Chem. Inf. Model 2010, 50,349 . 